\title{
FT-IR Application for the Detection of Pistachio Oil Adulteration
}

\section{ALI SHEIBANI*, NASER GHOTBADDINI-BAHRAMAN and FATEMEH SADEGHI}

\author{
Department of Chemistry, Yazd Branch, Islamic Azad University, Yazd, Iran. \\ ${ }^{\star}$ Corresponding author E-mail: sheibani@iauyazd.ac.ir
}

http://dx.doi.org/10.13005/ojc/300335

(Received: May 10, 2014; Accepted: June 18, 2014)

\begin{abstract}
In this work, fourier transform infrared spectroscopy (FT-IR) is used to identify and detection the adulteration of pistachio oil with cheap edible oils of corn, sunflower and soybean. For this purpose, pistachio oil was blended with cheap oils at concentration level of 10 to $60 \%(\mathrm{w} / \mathrm{w})$. Then, FT-IR spectra of pure and adulterated pistachio oil samples were obtained. The fingerprints region was found to be useful in investigation of the adulteration of pistachio oil. At this region, the absorbance peaks of FT-IR decreased by increasing the adulterant amount with a linear relation that can be applied for the quality and quantity purposes. The obtained results showed that the proposed method can be considered and used as an alternative method in the detection and semi-quantization of adulteration in pistachio oil.
\end{abstract}

Key word: Pistachio oil; Adulteration; FT-IR.

\section{INTRODUCTION}

Analysis and control of the quality of edible oils is an important subject in most of the countries. Oils are complex mixtures containing a wide range of compounds such as di and tri-acylglycerrols, free fatty acids, phospholipids and other components. The adulteration of high cost oils with cheaper oils is a serious matter because there is a great price difference among them and also may cause harm to the health of consumers ${ }^{1-3}$. Among, numerous articles have been reported to detection or determine the adulteration olive oil with various seed oils ${ }^{4,5}$.
Pistachio oil is also an expensive and important product that has a good market and can increase the added value of pistachio cultivation. Pistachio is one of the most significant oil seeds from the family of Anacadicea. It is composed of a kernel and a husk. The husk has a hard layer named shell and a softer layer called hull. The kernel has a green skin with a sweet taste and occupies different portions of the kernel in various types of pistachio. Compared to other nut oils, pistachio oil has a particularly strong flavor. Pistachio oil is rich in unsaturated fatty acids and vitamin $E$ and also is useful for health. It contains $12.7 \%$ saturated fats, $53.8 \%$ monosaturated fats, $32.7 \%$ linoleic acid and 
$0.8 \%$ omega-3 fatty acid. Pistachio oil is used as a table oil to add flavor to foods. It is also used in skin care products ${ }^{6-8}$. Therefore, adulteration of it involving the replacement with lower grade and cheaper oils can be performed.

Several techniques were proposed to control and detect the adulteration of edible oils. HPLC, GC, UV, NMR, Chemiluminescence and Fiber optic sensor have been widely used for this purpose ${ }^{9-14}$. Fourier transform infrared spectroscopy (FT-IR) is a technique used in the food product adulteration area or quality control. It is a rapid, nondestructive technique with short time for the sample analysis. FT-IR can be also used for quantitative analysis purposes. The intensity of bands in the spectrum is proportional to the concentration of the studied compounds ${ }^{15-18}$.

In present study, FT-IR is developed for the detection of pistachio oil adulteration with other edible oils including: corn, sunflower and soybean. Based on results and also because of simplicity and low cost, FT-IR can be applied as an alternative analytical method for the pistachio oil adulteration.

\section{EXPERIMENTAL}

\section{Materials and Methods}

Pistachio samples were obtained from Rafsanjan area of Iran. The pistachio kernel was ground and its oil extracted using Soxhlet procedure for $3 \mathrm{hr}$ with $\mathrm{n}$-hexane as the extraction solvent. Corn, sunflower and soybean oils were purchased from local supermarkets. Chemicals and solvents used in this work purchesd from Merck (Darmstadt, Germany).

FT-IR spectra of the oil samples were recorded on a Bruker Tensor-27 spectrometer Different mixture samples (w/w\%) of various kinds of vegetable oils with pistachio oil were prepared. 5 $\mu \mathrm{L}$ of pure and blended pistachio oils was deposited and spread as homogenously and one layer between two plates of $\mathrm{NaCl}$ crystal. For obtain the good results, this step is important. The prepared samples spectra (FT-IR) were recorded within 4000 to $400 \mathrm{~cm}^{-1}$. The peaks height in the wavenumbers of 1094,1116 and $1164 \mathrm{~cm}^{-1}$ were considered and used in the adulteration study of pistachio oil. The equations for study of the pistachio oil adulteration in blended samples were obtained by least squares linear regression.

\section{RESULTS AND DISCUSSION}

The first part of this study involved structurally differentiating between pistachio oil and the mixture of it with mentioned cheap oils and then detecting and determining the adulteration of pistachio oil. For this purpose, the spectra of pure pistachio oil were obtained and compared with the spectra of cheap oils and also pistachio oil blended to them.

Table 1: The equations and correlation coefficients obtained of pistachio oil mixed with different ratios of corn, sunflower and soybean oils in various wavenumbers $\left(\mathrm{cm}^{-1}\right)$.

Corn

\begin{tabular}{lccc}
\hline Wavenumber $\left(\mathrm{cm}^{-1}\right)$ & 1094 & 1116 & 1164 \\
Equation & $\mathrm{Y}=-0.0013 \mathrm{X}+0.39$ & $\mathrm{Y}=-0.0024 \mathrm{X}+0.37$ & $\mathrm{Y}=-0.003 \mathrm{X}+0.51$ \\
$\mathrm{R}^{2}$ & 0.9620 & 0.9501 & 0.9742 \\
Sunflower & & & \\
Wavenumber $\left(\mathrm{cm}^{-1}\right)$ & 1094 & 1116 & 1164 \\
Equation & $\mathrm{Y}=-0.006 \mathrm{X}+0.75$ & $\mathrm{Y}=-0.0058 \mathrm{X}+0.73$ & $\mathrm{Y}=-0.0064 \mathrm{X}+0.89$ \\
$\mathrm{R}^{2}$ & 0.9509 & 0.9320 & 0.9476 \\
Soybean & & & \\
Wavenumber $\left(\mathrm{cm}^{-1}\right)$ & 1094 & 1116 & 1164 \\
Equation & $\mathrm{Y}=-0.0027 \mathrm{X}+0.42$ & $\mathrm{Y}=-0.0027 \mathrm{X}+0.43$ & $\mathrm{Y}=-0.0037 \mathrm{X}+0.58$ \\
$\mathrm{R}^{2}$ & 0.9681 & 0.9517 & 0.9591 \\
& & & \\
\hline
\end{tabular}


Table 2: The recovery results obtained using the proposed method in detection and semiquantization of pistachio oil adulteration

\begin{tabular}{lccc}
\hline Sample & Added (\%) & Found (\%) & Recovery (\%) \\
\hline 1 & 15 & 13 & 87 \\
2 & 20 & 22 & 110 \\
3 & 35 & 31 & 88.6 \\
4 & 40 & 43 & 107.5 \\
5 & 60 & 55 & 92 \\
\hline
\end{tabular}

FT-IR spectra of the edible oils show that there is a notable difference in the spectral region between $1300-1000 \mathrm{~cm}^{-1}$, so that the oil composition affects the position of band and its intensity ${ }^{5}$. This is found to be very useful in detecting the edible oil adulteration. Figs. 1-3 show the FT-IR spectra of the pure and mixed pistachio oil with different ratios of corn, and sunflower soybean oils. The

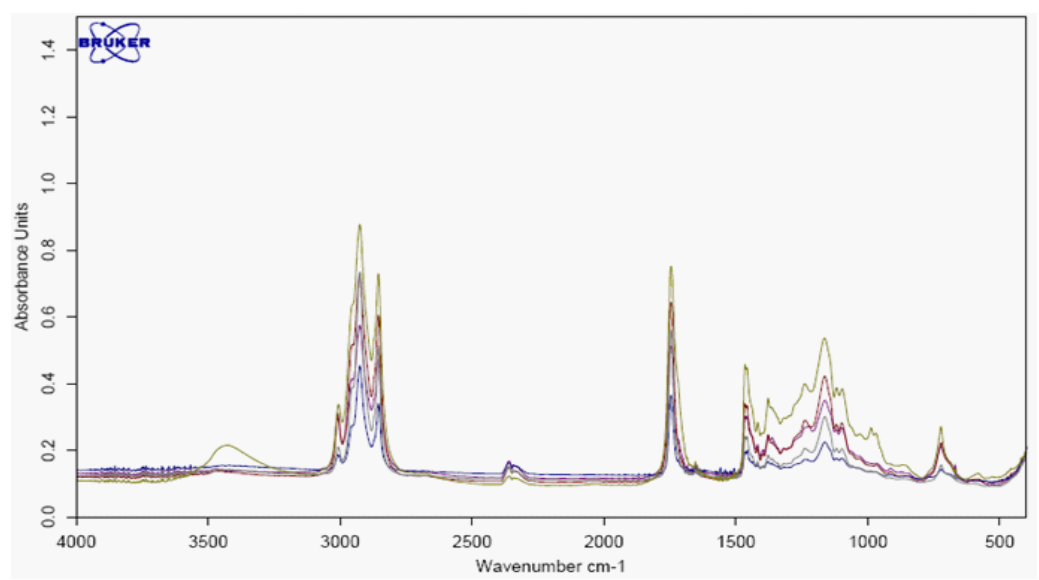

Fig. 1: FT-IR spectra of pistachio oil (top) mixed with different ratios $(25,50,75$ and 100$)$ of corn oil

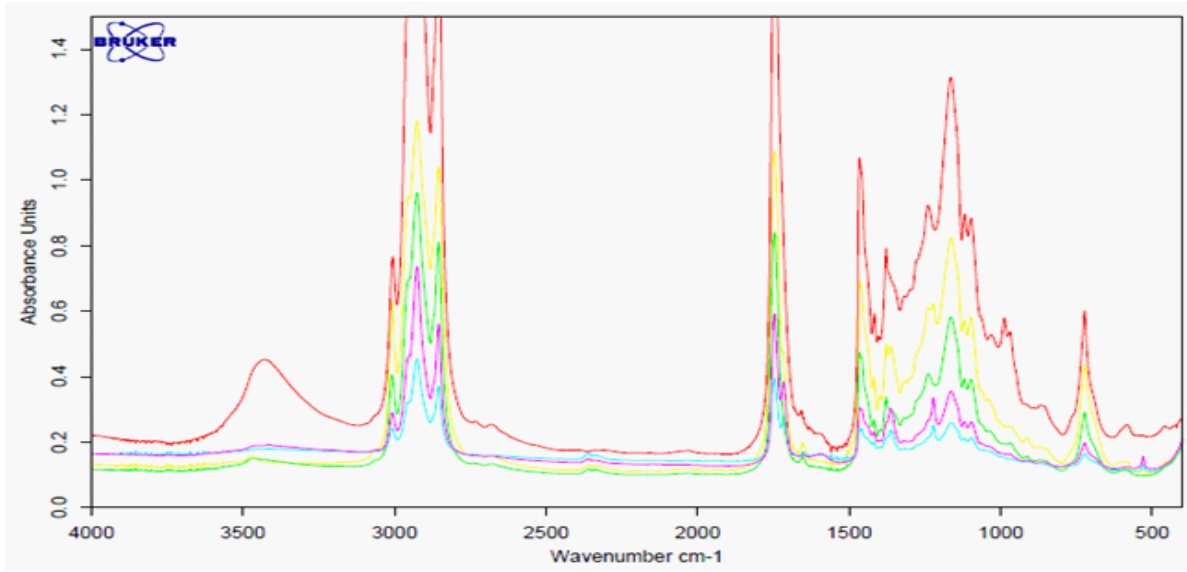

Fig. 2: FT-IR spectra of pistachio oil (top) mixed with different ratios $(25,50,75$ and 100$)$ of sunflower oil 
intensity of the absorption peak at 1094, 1116 and $1164 \mathrm{~cm}^{-1}$ decreases by increasing the amount of adulterant oils. Based on this, the percentage of the added edible oil to pistachio oil was determined. Table 1 shows the equations of change in the peak height in the mentioned wavenumbers with various percentages of cheap edible oils. Correlation coefficients have also been reported that shown a good linear relation between them. The lowest correlation coefficient $\left(0.9320\right.$ at $\left.1116 \mathrm{~cm}^{-1}\right)$ is observed for adulteration with sunflower oil while the best results $\left(0.9742\right.$ at $\left.1164 \mathrm{~cm}^{-1}\right)$ are seen for corn oil.
In order to evaluation of the proposed method, different amounts of the cheap edible oil were added to pistachio oil and the adulteration percentage was calculated. The recovery results were given at Table 2, showed a good agreement between the added and found amounts of cheap oils for the adulteration pistachio oil study. According to results at this Table, the proposed method can successfully be applied to the detection and semiquantitative assignment of pistachio oil adulteration. It can also perform to the development in food control and analysis.

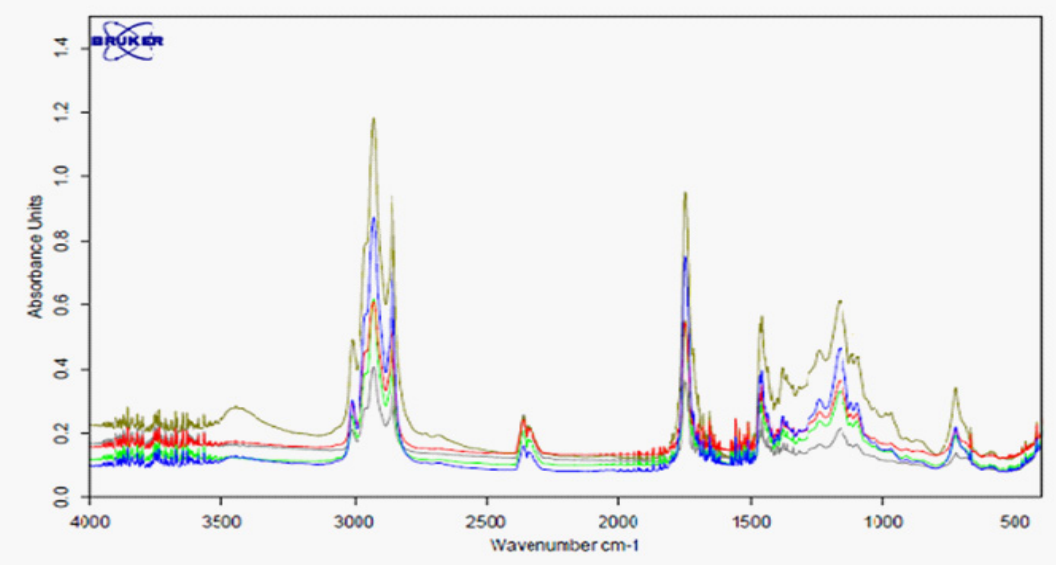

Fig. 3: FT-IR spectra of pistachio oil (top) mixed with different ratios $(25,50,75$ and 100$)$ of soybean oil

\section{CONCLUSION}

In summary, the present work developed the FT-IR application for the monitoring and detecting of the adulteration of pistachio oil with the lower priced edible oils. The absorbance changes with increasing the amount of adulterant oil were used for the detecting of adulteration. The results prove the validity and capability of the proposed method in detection of the pistachio oil adulteration.
Adulteration as low as $10 \%$ could be detected rapidly using FT-IR. The study leads to the conclusion that the proposed method can be potentially considered as an alternative procedure in the quality control of pistachio oil and other expensive oils.

\section{ACKNOLEDGMENT}

The authors would like to acknowledge the IAU. Yazd Branch, for financial support of this work.

\section{REFERENCES}

1. Rohman, A.; Cheman, Y. B. J. Food Lipids 2009, 16, 618-628.

2. Obeidat, S. M.; Khanfar, M. S.; Obedat,W. M. Aust. J. Basic Appl. Sci. 2009, 3, 2048-2053.
3. Özdemir, D.; Özturk, B. J. Food Drug Anal. 2007, 15, 40-47.

Cserháti, T.; Forgács, E.; Deyl, Z.; Miksik, I. Biomed. Chromatogr. 2005, 19, 183-190. 
5. Allam, M. A.; Hamed, S. F. J. Appl. Sci. Res. 2007, 3, 102-108.

6. Sheibani, A.; Ghaziaskar, H. S. LWT- Food Sci. Technol. 2008, 41, 1472-1477.

7. www. hsph. harvard. edu/nutritionsource.

8. www. en. wikipedia. or/wiki/pistachio oil.

9. Cordela, C.; Moussa, I.; Martel, A. C.; Sbirrazzuoli, N.; Lizzani-Cuvelier, L. J. Agric Food Chem. 2002, 50, 1751-1764.

10. Andrikopoulos, N. K.; Giannakis, I.; Tsamtzis, V. J. Chrom. Sci. 2001, 39, 137-145.

11. Mavromoustakos, T.; Zervou, M.; Bonas, Z. G.; Coulouris, A.; Petrakis, P. J. Am. Oil Chem. Soc. 2000, 77, 405-411.

12. Papadopoulos, K.; Triantis, T.; Tzikis, C. H.;
Nikokavoura, A.; Dimotikali, D. Anal. Chim. Acta 2002, 464, 135-140.

13. Libish, T. M.; Linesh, J.; Bobby, M. C.; Biswas, P.; Bandyopadhyay, S.; Dasgupta, K.; Radhakrishnan, P. Optoelectron Adv. Mater. 2011, 5, 68-72.

14. Vlachos, N.; Skopelitis, Y.; Psaroudaki, M.; Konstantinidou, V.; Chatzilazarou, A., Tegou, E. Anal. Chim. Acta 2006, 573, 459-465.

15. Tay, A.; Singh, R. K.; Krishnan, S. S.; Gore, J. P. LWT-Food Sci. Technol. 2002, 35, 99-103.

16. Baeten, V.; Aparicio, R. Biotechnol Agron. Soc. Environ. 2000, 4, 196-203.

17. Zhang, Q.; Liu, C.; Sun, Z.; Hu, X.; Shen, Q.; Wu, J. Food Chem. 2012, 132, 1607-1613. 